Review Article

\title{
A REVIEW ON APPLICATION OF NANOADJUVANT AS DELIVERY SYSTEM
}

\author{
HITESH KUMAR DEWANGAN*, SHUBHAM SINGH, ROHIT MISHRA, ROSHAN KUMAR DUBEY
}

Institute of Pharmaceutical Research (IPR), GLA University, Mathura, NH-2, Mathura Delhi Road, Chaumuhan Mathura, Uttar Pradesh (India), 281406

Email: hitesh.dewangan@gla.ac.in

Received: 14 Jan 2020, Revised and Accepted: 11 Apr 2020

\section{ABSTRACT}

Worldwide immunization can save millions of peoples to lives year by using the vaccines. The subunit of antigen components is manufactured which can stimulate the immune system by providing specific immunity against specific diseases. Subunit vaccines have many advantages like as high safety profile but having limited ability to provide immunogenicity. These traditional subunit vaccines activate only innate immunity, encourage cell-mediated transport of antigen to lymphoid tissues. Newly nano-adjuvants based vaccines carrier systems like liposomes, virosome, micelles, polymeric particles, protein, and peptides are developed by using various substances like viral proteins, polymer and polystyrene having immanent adjuvanticity and also provide exalted capability in manufacturing subunit vaccines. It has chromospheres substances that have various properties such as targeted, anti-damaging and caliber to lead immune reactions towards Th1 and Th2 route, which is an important feature for humoral as well as cellular immunity. The whole thing based on the carrier system, the role of nano-adjuvants, its pharmacokinetics and distribution in the body system. It has the ability to provide antigen-specific immunity to both systemic as well as mucosal by different vaccination passage. Also, the nano-adjuvants based vaccine suggested that direct targeting of antigen to improve the vaccine potency without sacrificing safety.

Keywords: Nanodjuvants, Immune system, Carriers, Antigen, Nanovaccine

(C) 2020 The Authors. Published by Innovare Academic Sciences Pvt Ltd. This is an open access article under the CC BY license (http://creativecommons.org/licenses/by/4.0/) DOI: http://dx.doi.org/10.22159/ijap.2020v12i4.36856. Journal homepage: https://innovareacademics.in/journals/index.php/ijap

\section{INTRODUCTION}

The most adequate means of controlling infectious disease-related illness and death is the vaccination. The World Health Organization (WHO) estimated that over 2.5 million child deaths each year worldwide has been prevented by vaccination [1]. A vaccine is a biological preparation that improves immunity to a particular disease. The traditional types of vaccines contain either dead or liveattenuated microorganisms, inactivated toxins (Toxoid), protein subunits, or polysaccharide antigens or conjugates. Several innovative vaccines are in the development stage, such as recombinant vector and DNA vaccines $[2,3]$. WHO given data of illness averted cost and averted deaths of people in every year presented in fig. 1 .

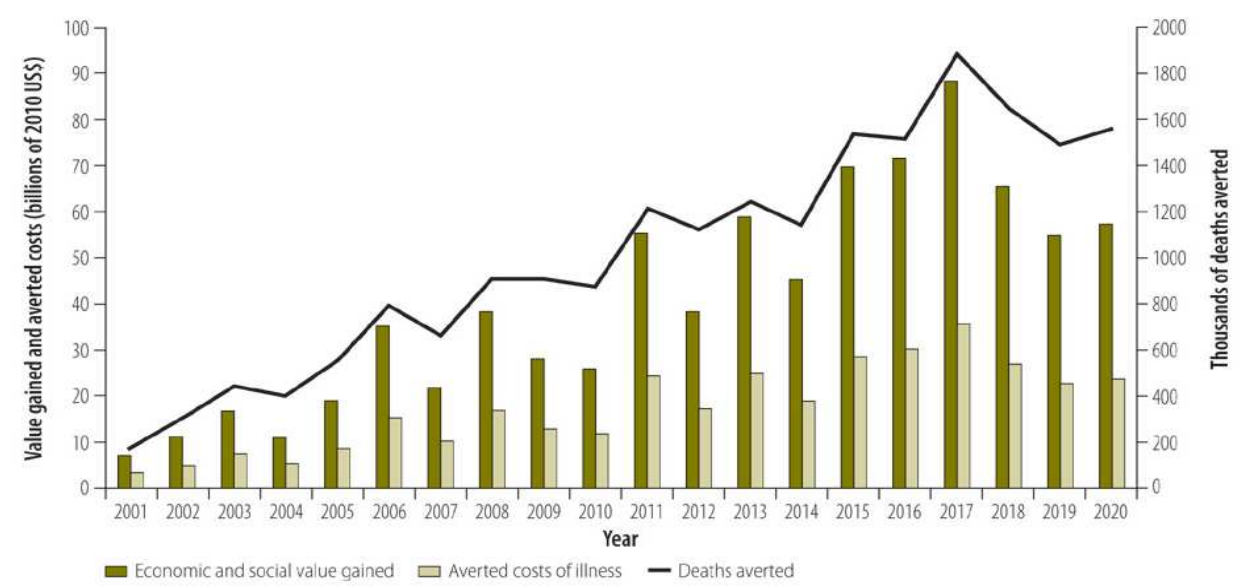

Fig. 1: Estimated economic impact of vaccinations in middle-income countries [According to Bulletin of WHO, Vol: 98, 2020]

These agents resemble a disease-causing microorganism and stimulate the body's immune system to recognize the agent as a foreign substance, destroy it, and "remember" it. Therefore, the immune system can more easily challenge these microorganisms upon subsequent encounters. Appropriate vaccine administration/delivery are the key challenges to ensure successful vaccination. Most of the vaccines are administered via the subcutaneous (SC) or intramuscular (IM) routes [4, 5]. Hypodermic injections are associated with pain and distress that might lead to patient compliance and require highly trained personnel for administration. They are associated with a risk of disease transmission due to the possibility of needle-stick injuries or re-use of contaminated needles $[1,6]$. Presence of dendritic cells
(DCs) at the administration site that takes up the antigen process it and presents it to T lymphocytes in the draining lymphoid organs, whereas subcutaneous fat and muscle tissue contain relatively few DCs. the dermis and the epidermis are densely populated by different subsets of DCs. Consequently, antigen delivery by hypodermic injection will bypass the skin's immune cells leading to less efficient vaccination. For this reason, the skin represents an ideal site for vaccine delivery $[1,7,8]$. Experts in the field describe recent innovations in the design, evaluation, and use of novel vaccine delivery devices and systems. In particular, they describe novel polymeric micro-needle systems for the delivery of DNA vaccines. 
The literature analysis of vaccine and nanoadjuvants carrier system carried out by surveying related peer-reviewed research articles from several online bibliography sources such as Scopus, Amazon, Google Scholar, Mendeley, Scienceopen, Springerlink, Research gate, Pubmed, and Shodhganga. Literature search libraries of the VaccineWorld Health Organization (WHO), the council for scientist and industrial research (CSIR) and Vaccines and Vaccine Manufacturing Institutes were also available. The web search strategy included keywords such as vaccine, adjuvant, nanoadjuvant, nanocarrier, immune system, nanovaccine, etc. I also performed a quantitative article review in the year of 1980-2020, based on the descriptions and abstracts, database records and other sources. Only certain studies that documented the application of nanoadjuvant and vaccine were included. In this review, articles discuss the delivery of vaccines with the help of a nano-adjuvants carrier system. The development of vaccine nano-adjuvants and the emerging area of nano-adjuvants based therapies are also discussed in detail $[9,10]$.

\section{Role of adjuvants in the vaccine system}

In the 21st century, vaccines are facing major population-related challenges. As such, the prevention of these infectious disease adjuvants had been used. Nowadays, a successful vaccine has not been possible using traditional technologies. It is minimizing the poor immunogenicity of the vaccine by using purified sub-unit antigen as well as increase the duration of action. Some of the vaccines require additional adjuvants for improving the quality of immune response. By improving the adjuvant immune response in the large population where reduced prototypical vaccine response, such as elderly and infants. To the initiation of the signal of the innate immune system, the type of adaptive immune response to delivered antigen by the choice of adjuvants for the activation of the specific T cell response [3]. The normal immune system generation process is presented in fig. 2. Development of the nano-adjuvants which contain more than one immune stimulatory molecules like the development of malaria vaccine.

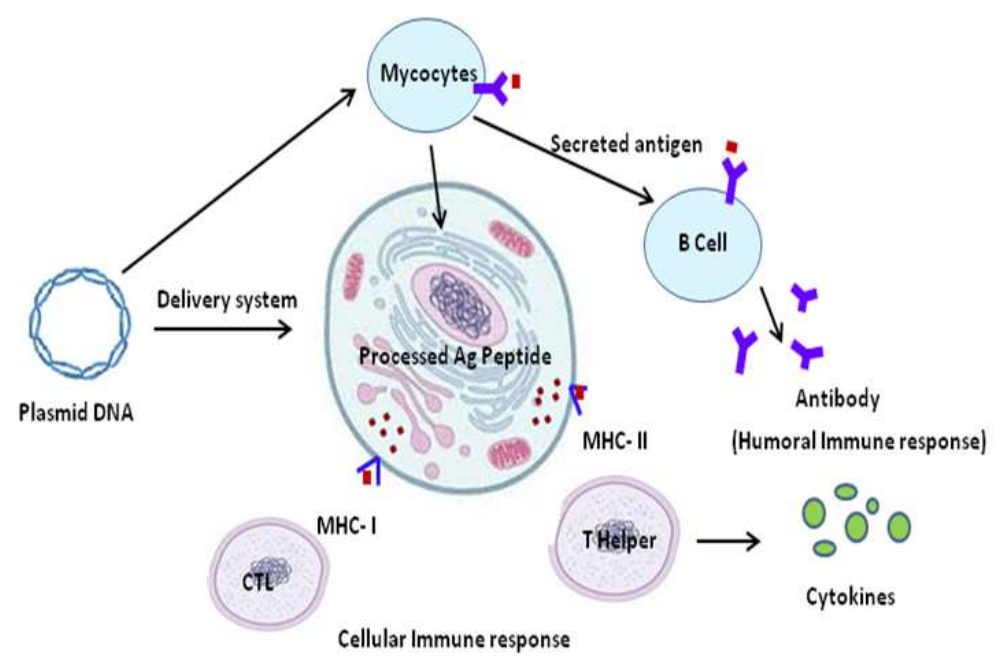

Fig. 2: Schematic representation of immune system mechanism [15]

In mid-1980, a suitable antigen was recognized by vaccine development. Aluminum is used as adjuvants that fail to provide and pre-clinical and human challenges studies were regulated. Also, explain different combinations of immune-stimulatory molecules. In the mid-1990, other than aluminum the use of first vaccine Hepatitis A was licensed which use a virosome as an adjuvant system. Aspherical phospholipid layered structure of the virosome and component can be varied to direct and uptake and intact with innate immune response. In the last $20 \mathrm{y}$, six more adjuvant has been included in the licensed vaccine. The most customary administered adjuvant is aluminum. It is used in oil in water emulsion, in which oils are improved reactogenicity in the compression of frond's adjuvants. The oil in water emulsion is the example of the squalene, which is naturally and metabolized oil. These types of emulsion induce a humoral and cellular immune response. For the enhancement of $\mathrm{T}$ cell immune response, some of the adjuvants is used in combination. For example, Pandemic influenza vaccine H5N1, which improve the immunogenicity in human. Without adjuvant influenza vaccine shows lower immunogenicity. By the compression of the non-adjuvants and adjuvants H5N1 Pandemic influenza vaccine-induced and increased the immunogenicity in all age groups and antigen sparing, allowing increased supply and cross-reactive immunity sub-clade variants. Due to the addition of adjuvants provide the benefits and confirmed the H5N1 experience $[3,11]$.

Pandemic influenza vaccine is entitled advancement; new adjuvants have developed the vaccine against some challenging pathogens such as HPV (Human papillomavirus). By the immune system, the undetected HPV, it is required that the high amount of the antibody levels for achieving the optimum concentration in the cervix, where the infection is done. Enhancement of antibodies with the aluminum adjuvant formulation that, has been inducing the HPV specific antibodies by the HPV-16/18-ASO4-Adjuvant vaccine. HPV-16/18ASO4 vaccine is licensed during the use in the conclusive demonstration efficacy. Show in the poor vaccination in the population by the improve immune response. For example, compression with the healthy individual patient are less tend to respond to the aluminum-adjuvant hepatitis $B$ vaccine, but in the case of the acute hepatitis B vaccine, more risk occurs. Licensed ASO4 adjuvants for the Hepatitis B vaccine show the higher and more duration antibody response is compared to the aluminum adjuvants hepatitis $B$ vaccine, at the last stage of the renal disease increase the cellular response in the patients $[2,12]$.

The rapid advancement of the development of the vaccine is shown by the understanding and potential role of the adjuvants. In a large population, the development of the new targeted adjuvants vaccine is challenging for pathogens such as dengue fever, cytomegalovirus infection, virus, HIV, Lung cancer and melanoma in malignancies. Immediate improvement in the antigen and adjuvants design, the remaining gaps knowledge in our understanding of the immune system; such as simultaneous contribution in the adaptive and innate response against individual's pathogens. Some of the biological tools, such as vaccinosis, transcriptomia, and epigenetics, are contributing to our understanding of vaccination and immunity. With the help of the mode of action of the adjuvants evaluate the safety and efficiency of new adjuvants $[7,13,14]$.

\section{Adjuvants approved for human vaccine}

\section{Aluminium salts (Alum)}

By the utilization of the suspension of alum precipitated Diphtheria toxoid, submit the data regarding the aluminum compound in 1962. 
Aluminum salt is mostly used in the human vaccine. For stimulating the Th2 immunity, these adjuvants are used till more than $80 \mathrm{y}$, in the commercial vaccination. In the human vaccine such as hepatitis A virus (HAV), Human papillomavirus (HPV), hepatitis B virus (HBV), diphtheria, and meningococcal, the component of alum are mostly used.

In the alum some advantages such as long-lasting antibody titer, high augmentation, antigen stabilization, and high safety record. However, adjuvants (alum) have not the capacity to screening the cytotoxic $\mathrm{T}$ cell and which vaccine containing alum adjuvants cannot be sterilized by frozen or lyophilized and filtration. Some adjuvants are very effective but these are not used because these are toxic. The mechanism of action is not well identified but these are used (widely) in the licensed human vaccine. In 1925, Glenny gives the mechanism of alum, which is well known as "depot effect," and the depot is observed at the injection site where continuously releasing the antigen. Some controversy in the depot formation and the recent studies find out that the alum adjuvant is not required in the depot formation $[3,15]$.

\section{MF59 and adjuvant system 03 (AS03)}

In Europe, MF59 approved for the H1N1 influenza vaccine and the H5N1 pandemic influenza vaccine. With the help of the dendritic cells (DCs), these adjuvants are activated CD4 T cells and uptake by the MF59. It produced high antibody titers by the MF59 with the balance IgG1: IgG2a responses. The evaluation of the MF59 with human immunodeficiency virus (HIV) and cytomegalovirus (CMV) vaccine are under clinical trials. Squalene oil is present in both MF59 and ASO3, and they have different compositions. Alpha-tocopherol has been present in the ASO3, which is responsible for the nonspecific activation of the immune system in mice [2].

\section{Monophosphoryl lipid A (MPL) and adjuvant system04 (AS04)}

ASO4 adjuvants vaccine is licensed for human use. This adjuvant used in the HBV vaccine (Fendrix) for Haemodialized patients and the HPV vaccine (Cerarix) for papillomavirus. MPL adjuvants are used for cancer. This adjuvant interacts with TLR4 and activates innate immunity. It leads to the activation of NF- $\kappa B$ signaling and the production of pro-inflammatory cytokines and chemokines. CCL2 and CCL3 (chemokines) enroll monocytes and macrophages and at the injection site activate the dendritic cells (CDs). After the maturation and migration to the lymph nodes, stimulate the cell-mediated immunity (CMI) by the interaction with the T cells by the DCs. Finally, it triggers the adjuvant effect on antigen-presenting cells (APCs).

Transmembrane signaling protein contains the toll-like receptor (TCR), comprising the pattern recognition receptor (PPR). These adjuvants are already licensed for a human vaccine. Immune stimulatory adjuvants TLR (agonist) which activate TLRs. Based on the use of the TLR agonist, some advancement in the design of adjuvants. Salmonella Minnesota R595 strain produces the chemically detoxified derivatives, which are the antagonist of the TLR4 agonist [7]. Generation of Th1 immune response increases by increasing the production of pro-inflammatory cytokines such as IL2 and IFN- $\gamma$, with the help of increasing the MPL $[16,17]$.

\section{Immunization}

Oral immunization of vaccines is mostly applied for the intestines, which have several complications and decades. Now a day, a very little mucosal or intestinal vaccine is available in the market. Oral or mucosal vaccine is the easiest technique for immunization, which is completely preventing any mucosal infection. Here, present the various approaches and technologies for oral immunization [18]. Most of the human pathogens enter the body through mucosal surfaces. It is necessary for the development of vaccines, adjuvants or delivery systems, which is effective for mucosal immunization with highly desirable. Most of the vaccines available today are given parenterally due to the problems in achieving effective mucosal immunization [8]. The major problems in developing effective mucosal vaccines include.

1) Instability of the antigen when administered by orally such as unfavorable conditions of $\mathrm{pH}$, presence of bile salts and enzymes such as proteases.
2) Induction of tolerance for soluble antigens delivered in mucosal surfaces.

3) The inefficiency of uptake of antigens by mucosal surfaces.

4) Interference by maternal antibodies obtained through breastfeeding.

In recent years, biodegradable polymer microspheres have been used for effective mucosal immunization in animals [19, 20].

\section{Bio-distribution of nano-adjuvant}

Lymphatic vs cell-mediated transport for vaccine and nanoadjuvant delivery to lymph nodes

$\mathrm{B}$ and $\mathrm{T}$ cells recirculate through the spleen, lymph nodes (LNs) and antibiotics. It has been accessible to those organs that physically to generate an immune response. For this purpose, the nano-adjuvant plays an important role. Vaccine transactions to LNs can be mediated by lymphatic drainage or immune cell-mediated transportation from an injection site. The adjuvant first generation (aluminum salts, aluminum, MF59), works by promoting cell migration to intramuscular injection sites. Then, the antigen vaccine is internalized and carried by neutrophils, monocytes, and DCs into the draining of $\mathrm{T}$-cell responses [21-23]. The $\mathrm{T}$-cell response is supported by antigenic nano-adjuvants. The intramuscular injection place also drains antigen; Since MF59 is not associated directly with antigens, not all antigens are stored at the alum injection site, MF59 can also promote antigen retention in LNs which probably supports B cell-mediated reactions [24, 25].

The intramuscular path is long-supported as these nano-adjuvants can cause important reactions in the injection site by administration with alum or oil in water emulsions. Other locations, such as subcutaneous, have promoting solid, safe and best humoral reactions for many vaccines and nano-adjuvants [26].

However, in the majority of cases, APC trafficking LNs vaccines have been shown to account for small populations (e. g. $0.1-5 \%$ of the total LNs). Thus, in traditional muscle-draining LNs, a minute amount of antigen is typically detected. It has limited strength of the immune response in ways that could be of importance for intracellular pathogens $[17,27]$.

Alternatively, a route like subcutaneous or intradermal injection is effective and in combination with formulation to minimize the injection site reactions. There are now several methods that allow vaccines to build up from these locations in elevated concentrations in LNs and to reach a majority of DCs in nodes. Particularly, molecular weight and its measurement are directly related to the lymphatic drainage system. But for big macromolecules, transport is extremely ineffective in the basement membrane and tight interconnections of the endothelial cells [28, 29].

Thus, lymph uptake and molecular weight are virtually linear up to about $45 \mathrm{kDa}$. Over which proteins are almost 100 percent trafficking in animals and human beings. This relation between molecular dimension and absorption of the lymph describes by lowmolecular adjuvants. Examples such as imidazoquinolines have elevated systemic exposure or inflammatory toxicity after parenteral injections. Efficient lymphoid tissue trafficking is only an early phase; LNs must also capture vaccine products later on. A second path to systemic exposure to antigens and nano-adjuvants is the material that flows across the lens into systemic circulation by the thoracic pipeline. Study of tiny (30 nm in diameter) PEGylated polymer particles, which achieve higher levels in the blood similar to intradermal injection in mice $[21,22,25]$.

\section{Targeting antigen-presenting cells in lymphoid organ}

Antigens or nano-adjuvants have intended to help the accumulation of LNs by targeting immune cells or endogenously protein. For instance, changes to oligonucleotide nano-adjuvants or peptide antigens with lipid tails intended to bind albumin have shown to support significantly increases the absorption of LNs. Binding to albumin prevented the dissemination into the blood of the lowmolecular-weight vaccine components. The efficiently trafficked 
them to the lymph nodes leading to accumulation in multiple DCs subsets that are residents in LNs. The improved accumulation of LNs resulted in a significant increase in immunogenicity relative to soluble antigen $[30,31]$.

DCs themselves are a heterogeneous collection of the cells consisting of many phenotypes with distinct immune response functions. For example, $C D-11 \mathrm{~b}+\mathrm{D}$ CDs play a significant role in priming $\mathrm{CD}-4+\mathrm{T}$ cells. The more effective antigens acquired from the extracellular environment are in the mice (CD-141+CC-1+CAD-1+DC in humans). Another approach to improving LNs absorption and vaccination efficacy is targeted at particular DCs subsets through the operation of vaccines with DC-binding ligands [32].

When the antibodies combined with antigens, nanoparticles (nanoadjuvants) and lentiviral vectors have to increase the LNs accumulation. The immunogenicity has been shown to improve vaccines that target the DEC-205 (CD205) or other cell surface molecules. These vaccines promote both the humoral and cellular immunity in humans. Thus, DEC-205 antibodies serve as proof of the many DCs targeting agents for vaccine administration that are being studied. However, the best way to achieve a vaccine is effectively targeted to deliver a specific subset of DCs is the involvement of nano-adjuvants in formulations [33, 34].

\section{Adjuvant effector properties and pharmacokinetic}

The formulation strategies to improve the delivery of nano-adjuvant loaded vaccines to LNs can often reduce the distribution as well as systemic toxicity. Some of the micelle-shaped lipids or aggregates that interact with proteins or reorganize based on the composition of the environmental solution and produce poorly defined products. The integration of these amphiphiles in the synthetic or multilamellar liposome bilayers allows, however, the generation of stable adjuvant nanoparticles with well-defined in vivo biodistribution characteristics [16, 30, 35, 36].

The AS01B/E adjuvant formula from GlaxoSmithKline, consisting of liposomes have monophosphoryl lipid A. The plants based Capone QS-21, is a proven liposomal adjuvant in many clinical studies, especially in malaria. The encapsulation of small molecular nanoadjuvants in nanoparticles or liposomes in polymers has shown that systemic exposure to those compounds that are eliminated [37]. SMIPs (tiny molecule immune potentiator) TLR7 agonists with aluminum binding phosphate groups were lately produced. When blended with alum, become linked to the adjuvant, concentrating on their action in LNs, muscle and muscle drain. Direct changes to the tiny molecules have also been used to change additive pharmacokinetics. The systemic distribution or toxicity of aluminum binding SMIPs was considerably less than the non-aluminum binding parents. While at the same time showing much greater efficacy for the promotion of humoral immunity in mice [38, 39].

\section{Programming pharmacokinetics of nano-adjuvant and vaccine}

\section{Nano-adjuvant artificial vs natural immunizations}

Chronic infections cause continuously elevated concentrations, such as IL-1,IL-6, TNF- $\alpha$, case-1, and type-IFN, which lead to serious immune-response defects. In comparison, most viruses infectious are frequently removed for seven days during "acute" bacterial diseases. But residual viral/antigenic can often be found in the space of four to $8 \mathrm{w}$ with a clear immune response. The length and extent of the exposure of the antigen and adjuvant during naive lymphocytes or memory cell boosts are considered to be an important factor for the degree of clonal development. The studies investigating the role of temporary dose profiles by repeated daily antigen/adjuvant an injection shows that over a week, exponentially increasing doses of antigen. Incorporation of adjuvants could significantly increase $\mathrm{T}$ cell response $[23,40]$.

The information shows that nano-adjuvants and vaccine exposure kinetics control strategies can have a significant positive impact on immunization reaction. Unformulated soluble antigens or adjuvants have flushing quickly over drainage by lymph fluxes within hours. Classical adjuvant products such as aluminum and oil-in-water emulsion (nano-adjuvant based) have a general belief that they produce antigen depots at the injection site. It also suggests that current vaccines may already reach a certain level of lasting vaccine exposure. However, the MF59 oil-in-water emulsions have not shown to alter the clearance or persistence of antigen in draining LNs (lymph nodes) at the injecting sites. In the case of alum, it seems to be a storehouse with some but not other antigens and is probably deorbited under in vitro circumstances by some of the antigens. Recent studies have shown that the injection site can be removed operatively after $2 \mathrm{~h}$ of injection, without any influence on the human immune response. The alum immunization suggested that the formation of depots is not a critical component of an alum action system [40-42].

\section{Nano-adjuvant and vaccine kinetics}

The non-biodegradable poly (ethylene-co-vinyl acetate) implants could release protein antigens in vitro in mice for up to one month. It leads to having antibody reactions equal to numerous injections with Freund's adjuvant. One of the earliest research on the use of synthetic polymer matrix to achieve continuous biology release. In the 1990s, biodegradable PLGA microspheres for encapsulation and slow release of vaccine antigens were used in many groups to produce practical formulations for prophylactic vaccines.

The fact that no biodegradable polymers formulation of encapsulated vaccine antigens has yet to move into a sophisticated clinical trial. However, the drawback is antigen degradation within the acidic environment, difficulties of manufacturing in large scales, and challenges to show the superiority of such formulations over alum. Conversely, the PLGA formulation showed hazard-signal or tolerogenic. (Tolerogenic therapies, which based on the principle that inducing the semimature phenotype in dendritic cells and then exposing them to the target antigen has to be allowing antigenspecific induction of T-cell tolerance).

Compounds are an effective approach and influence the adjuvant kinetics in vaccination that observed in preclinical models. The formulation of antigens and danger signals, which are implanted in the skin within porous PLGA polymer sponges are a second promising approach, particularly for the development of therapeutic vaccines. This PLGA scaffold-based vaccine approach has recently entered phase I clinical testing in patients in the case of melanoma $[40,43,44]$.

\section{Carrier system for vaccine delivery}

The parenteral inoculation, preparation, should be in the form of microspheres « 100 film so that it is injected with a syringe. For mucosal immunization or targeting the microspheres to antigenpresenting cells after parenteral inoculation, a size of $<10$ fold is desirable. The polymer used for the preparation of microspheres should be biodegradable, thus avoiding the need to remove the nondegradable polymer pellet or discs surgically after immunization. The formulation should be heat-stable, preferably lyophilized to avoid the need for a cold chain $[45,46]$. Biodegradable microspheres have also been evaluated for the controlled release of antigens and vaccines by parenteral immunization. Two broad approaches may be followed to achieve the controlled release of vaccines.

First is the pulsed release of vaccine antigens, which could be achieved by selecting polymers and encapsulation conditions. These types of microsphere have provided different rates of hydrolysis. The microspheres made up of two or more different polymers with varying rates of antigen release could be observed. The timedependent antigen release also has characteristics of microspheres $[47,48]$.

The second approach is the continuous release of an antigen for prolonged periods, which could result in the high levels of antibodies usually observed after multiple injections. In this approach, the characteristics of polymers affect the release profile of antigens. Depending upon the molecular weight and ratio of lactic to glycolic acid, PLGA polymers can have hydrolysis rates from a few days or weeks to several months or over a year. Several human vaccine antigens encapsulated in microspheres elicited antibody levels in animals similar to those obtained with adjuvants such as aluminum compounds and complete Freund's adjuvant $[49,50]$. 


\section{Lipid-based delivery systems}

Some of the earliest adjuvants and delivery systems developed took the form of lipid-in-water or water-in-lipid emulsions types.

Emulsions have been extensively tested in humans. MF59 is an immunologic adjuvant which is already approved for human, which used in squalene. An oil-in-water emulsion contains squalene, which drives humoral immunity and CD4+T memory cells. Micelles are one of the earliest examples of utilizing lipids as delivery vehicles. It consists of amphiphilic block copolymers displaying both hydrophobic and hydrophilic segments.

These unique properties drive their self-assembly into core-shell architectures which then allows for a payload to be delivered inside the core or attached on the outside to the shell. It is depending on the electrochemical properties of the vaccine formulation. However, micelles can often dissociate when diluted, leading to loss of the payload. Newer polymers allow for the intentional release of a payload in a controlled-release system. Micelles can also be synthesized in the nanometer range, which acts as delivery vehicles that can potentially penetrate the mucus layer and be taken up by mucosal antigen-presenting cells $[14,15,51,52]$.

\section{Liposomes}

Liposome is a simple microscopic structure in which the aqueous phase is entirely appended with the amphiphilic molecules such as lipid, phospholipids etc. The drug substances or molecules are enclosed or encapsulated or appended in the aqueous phase and the rate or extent of drug release depends on the physicochemical characteristic of lipids and phospholipids (fig. 3). For improving the immunity, macromolecules such as carbohydrates, lipids, proteins, and nucleic acids are appended for targeting of intracellular as well as extracellular. Liposomes are used for mucosal vaccination because it increases the stability of vaccine in the gastrointestinal tract. Such as the development of polymerized liposome, which has fused with the cell wall and quickly enters into the reticuloendothelial system $[53,54]$.

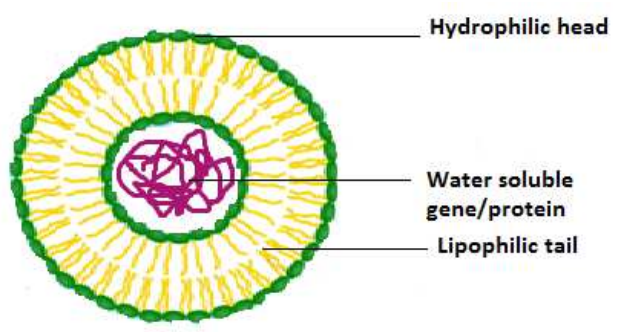

Fig. 3: Gene/protein incorporated liposome

A polymerized liposome is coated with specific types of molecules such as antibody filament, antibodies, and antigens. These are capable of binding with specific cell surface receptors or targeting sites. Mostly, liposomes are taken up by an antigen-presenting cell. Polymerized liposomes are used in vaccine delivery system because it allows for the cumulative ingested antigen to be processed and presented on MHC molecules. Liposome incorporating bile salts known as bilosomes also used in vaccine delivery in the influenza vaccine. These bilosomes are administered by the oral route. Liposome delivers the antigen preferentially to macrophages. Now a day's liposome is used for the treatment of HIV infection [55]. Liposome is increased the efficacy, reduces toxicity and protect against enzymatic degradation of antigen or drugs. For example, protect the molecules or drugs such as propranolol and cyclosporine from the external environment. Nowadays, liposomes used in the treatment of HIV infection [12].

\section{Emulsion}

Emulsion are heterogeneous biphasic liquid oral dosages form, normally $\mathrm{O} / \mathrm{W}$ or $\mathrm{W} / \mathrm{O}$ emulsion and multiple type emulsions such as $\mathrm{O} / \mathrm{W} / \mathrm{O}$ or $\mathrm{W} / \mathrm{O} / \mathrm{W}$ emulsions. In this carrier system, antigens are dissolved in the aqueous phase that will be emulsified in the oil phase by using the emulsifier. The sustained liberation of this system depends upon viscosity, a ratio of oil and water phase and droplet size. In the current scenario, emulsions are used for the vaccine delivery system. Most frequently, whereby $0 / \mathrm{W}$ type emulsion has more benefit as compared to W/O type emulsion because W/O type emulsion causes irritation and pain at the injection site $[5,56]$.

The $0 / \mathrm{W}$ type emulsions are suitable for vaccine transporter and increase the cytokine and chemokine environment to the antigenpresenting cell. For transport of vaccines into animal and human, immunization adjuvant are available like as salts of Al. But the use of these emulsion systems not required for additional adjuvants. $0 / \mathrm{W}$ type emulsions are widely used for the delivery of vaccines through the oral route. It provides immunity to the body, which protects against several types of micro-organisms. The advantage of an emulsion is to delivery of both types of drugs such as hydrophilic and lipophilic by increasing the solubilization $[8,12]$.

\section{Nucleic acid and peptide vaccine delivery}

PNA (Peptide nucleic acid) is a DNA mimic with a pseudo peptide backbone composed of $\mathrm{N}$-(2aminoethyl) glycine units with the nucleo-base attached to the glycine nitrogen via carbonyl methylene linkers. A brief overview of the history, properties, and application of PNA in drug discovery and DNA detection is presented. PNA was the first described in 1991 and has since then attracted widespread attention with the field of biology and medicine including drug discovery, genetics, diagnostic, molecular recognition, bioorganic chemistry, mechanical chemistry, physical chemistry, and molecular biology due to its chemical and physical property $[49,52]$. The structure features provide the DNA mimicking properties of PNA and give an overview of the structure backbone modification of PNA. PNA oligomers can form a very stable duplex structure with Watson crick complementary DNA, RNA or (PNA) oligomers. Particularly, about its efficient and sequence-specific binding to both single standard RNA and DNA as well as to double standard DNA. These are also bind targets in duplex DNA by helix invasion $[18,20,48]$.

The double helix of DNA is nature's simple and elegant solution to the problem of storing retrieving and communicating the genetic information of a living organism. DNA has many important characteristics' that allow it to perform these functions. Two of the most important properties are the specific and reversible nature of the hydrogen bonding between complementary base pairs. The properties which such as allow the strands of the double helix to be unwounded and then re-wounded in the same configuration. The field life science realized early on the important amplification of these traits. If specific, single strands DNA could be synthesized then the base sequence of the gene could be studied and manipulated these defined molecules. Nowadays, artificially synthesis of DNA or RNA is common. Synthetic oligonucleotides are now indispensable tools for life scientists, with many applications in molecular biology, genetic diagnostic and most likely also seen in medicine $[12,14,51]$. PNA may be used in many of the same applications as traditional synthetic DNA or DNA analogues. However, the greatest contribution of PNA may come from the development of new applications that cannot be performed using oligonucleotides $[56,57]$.

\section{Synthetic peptide vaccine design}

The solid-phase method is the most convenient and versatile approach for the direct synthesis of a peptide-antigen. The peptide antigens generate a macromolecule which has a high density of peptide antigen. This method utilizes a simple scaffolding of a low number of the sequential level (n) and trifunctional amino acid as the core matrix $2 \mathrm{n}$. The approach is called the multiple antigen peptide system (MAP). The MAP containing both core matrix and peptide of 9-16 amino acids and prepared in single synthesis by the solid-phase method. Six different MAPs specific antibodies found on rabbit and mice which produce antibodies that react with the corresponding native protein. In rabbit higher antibodies have hemocyanin as carriers. The approaches for the preparation of carrier bound antigen of predetermined and reproducible structure and might be suitable for generating vaccines [58-60]. 
Studied the effect of S. C. injection of a volume of FCA/antigen (0.5 $\mathrm{ml} /$ in rabbit) in one injection site compared to the same volume spread over 2 or 4 injection sites. Similar antibody responses were observed, for both immunization procedures. These results suggested that dividing antigen over more sites does not increase the antibody response. Administration of antigen at different routes like subcutaneous, intracutaneous, or intramuscular sites will lead to the slow release of the antigen and enhance the immune response. Always this slow and continuous release of antigen produces strong immune responses [13, 61].

Peptide and protein incorporated vaccine preparation some of the most dangerous epidemic infectious diseases could be efficiently controlled and to develop new ones to eventually control also those diseases for which no vaccine at yet available. Recombinant DNA methodology is used to express viral or parasitic protein in either bacteria, yeast or cultured animal cell in live vaccine vector $[62,63]$. A rational approach of identification and selection of protein segment prepared by synthetic peptide vaccine. It involves virulence factors that have to identify and characterization role in the infection mechanism with regard to immunological properties as many of the virulence factors known exhibit extensive antigenic variation [64]. The antigenic makeup of protein has been intensively investigation and accumulation evidence suggested through macromolecular antigen usually expresses multiple antigenic sites, anti-protein antibodies [65, 66].

\section{Niosomes}

Niosomes are made up of non-ionic surfactant vesicles that can encapsulate or entrapped both hydrophilic as well as lipophilic drugs. Lipophilic drugs are entrapped in the non-ionic surfactant region whereas hydrophilic drugs are entrapped or encapsulated in the aqueous core (fig. 4). Niosomes are more stable as a comparison to the liposome because it contains the non-ionic surfactant whereas liposome main constituents are phospholipids which will be affected by oxidation and temperature. Niosomes have versatile characteristics, low-cost vesicles used for the genes, drugs and vaccine transport. For the preparation of niosomes amphiphilic substances or molecules are required.

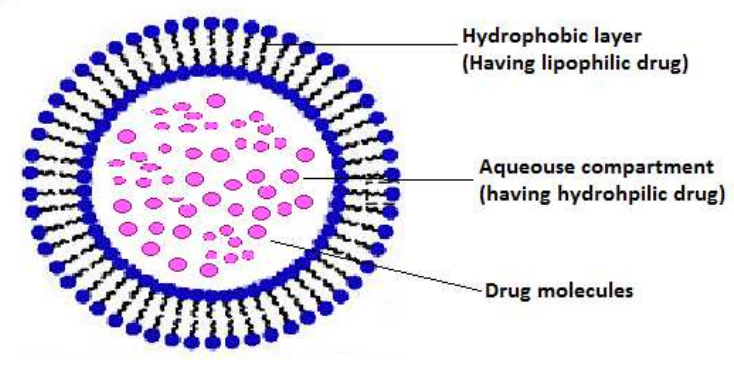

Fig. 4: Drug/protein incorporated niosomes

The amphiphilic substances contain the two parts, a hydrophilic head group which is also known as a polar head group and a lipophilic tail group which is also known as the non-polar tail group $[46,67]$. The development of safer, effective and new vaccines and their delivery are the most crucial aid to several researchers in all countries. Firstly brewer and Alexander introduced the use or application of niosomes for delivery of antigen, which gives immunity to Balb/C mice towards the BSA and said that niosomes have the best stimulator of Th-1 lymphocytes.

\section{Virosomes}

Virosomes are a high safety and efficacy technique for the delivery of medicaments. The technique is approved by the FDA for human use. Virosomes are applicable for the delivery of drugs, proteins, peptides, nucleic acids, antibiotics, etc. It increases the fusion activity or response in the endolysosomal pathway. Virosomes are used for vaccine or drug transport. It is consist of unilamellar or single macromolecules such as phospholipids membrane vesicles which absorb proteins earned from virus and make a fusion with targeted cells. Such macromolecules (virus-derived proteins) permit the virosomes transport system to fuse with the cells for immune activity. Result in the transport of their active or main constituent towards their targeted cells. After that, produce a specific reaction of the cell $[15,46]$. After the delivery of antigens, virosomes are decomposed into the body or in cells. The embedded virus proteins into the phospholipids double-layer not confirm only structural steadiness and likeness of virosomal preparation but also confirm the virosomes immunological behavior that will differ from other carrier systems such as liposome, lysosome, etc. Virosomes are vesicular transport or arrangement in which antigens are embedded into virosomes or adsorb on the floor of virosomes by hydrophobic interaction $[47,68]$. Virosomes are very biocompatible, biodegradable, non-toxic systems and there is no risk of disease transmission. The most important quality of virosomes is does not change in autoimmunogenity or anaphylaxis and it only has the efficiency to deliver the drug into the cytoplasm of target cells (fig. 5).

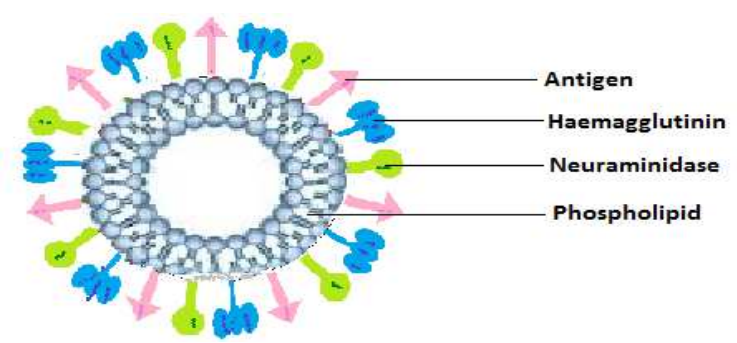

Fig. 5: Represent the various components of the virosomes

\section{Virosomes immunopotentiation mechanism}

The character evokes immunity to virosomes transport is dependent on the presence of epitopes, whether it will present on the floor or inside the virosomal transport or preparation in which PeviPRO are present on the floor and EviTER are present inside the virosomes. PeviPROgenerates humoral mediate immune response by breaking the antigens into endosomes cell and thus results in major histocompatibility complex-II antigen and also increase the cytotoxic T-cell activity [69].

Virosomes entrapment provides an appropriate demonstration of antigen by major histocompatibility complex-I due to fact the transport of antigen into the cytosol of antigen-presenting cells in an appropriate way. This new generation of vaccines has more advantages because it is impressive not only in Childs but also in immune-suppressed patients. They provide excessive protection to adsorb viruses because they are not replicating [70].

Membrane lipid and viral glycoprotein are part of the virus envelope and lack of genetic information. In Europe, for the influenza virus, the virosome vaccine is approved and In Asia, Europe and South America hepatitis A virus vaccine approved. Both vaccines utilize virosome derived from the influenza virus represented by immunopotentially reconstituted influenza virosome. The universal acceptable adjuvants for all age groups (children, adults and elderly) such as influenza virus (Inflexal V), both major histocompatibility complex (MHC-1) and MHC-2 contain the virosomal adjuvants, virosome are responsible for the CMI and humoral immunity both (fig. 6). Some advantages of Virosome vaccine are following

1) Protection of antigen from degradation,

2) High quality of long-lasting antibody response,

3) Suitability to the specific population such as infants, immunocompromised patients and the elderly,

4) Excellent safety profile. 


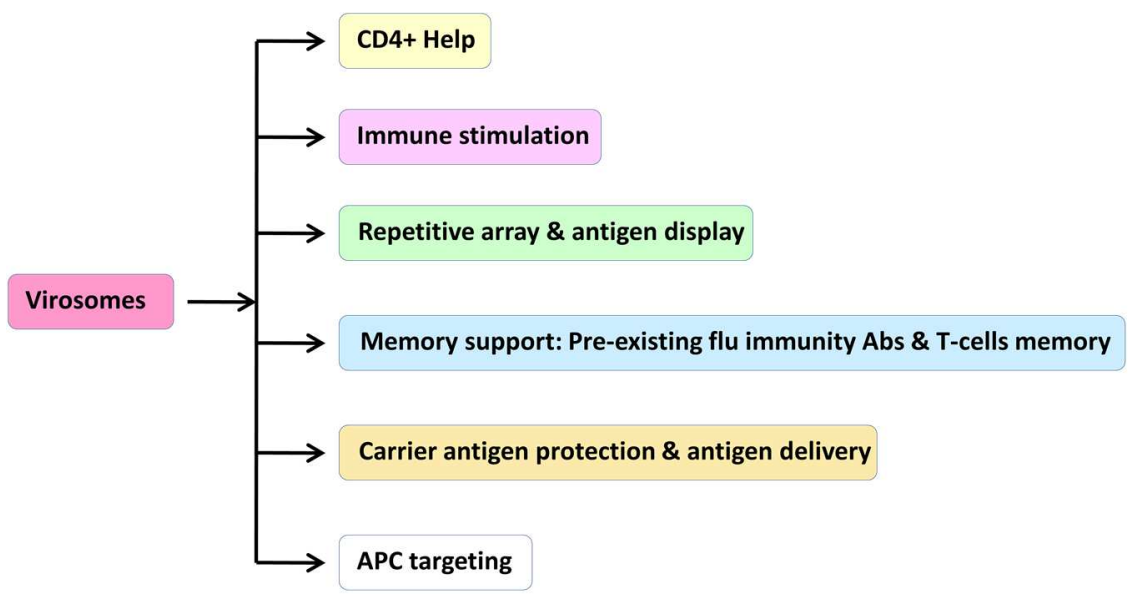

Fig. 6: Showing activation of antibody through virosomes

\section{DNA vaccine}

DNA vaccines are an effective substitute to alive or killed vaccines for the treatment of human and animal diseases such as allergy, autoimmune diseases, cancer disease, and other diseases that are fatal to humans and animals. Furthermore, the current invention in vector engineering and plasmid host strain enhanced plasmid, preparing quality and yield transfection efficiency, transgenic expression levels for a secure and very high-quality gene as a comparison to old generation vectors. Essentially, plasmid DNA vaccines consist of purified vectors that mix an eukaryotic vicinity-which consist of a strong enhancer/promoter for the expression of transgenic coding for antigenic/therapeutic proteins or peptides in mammalian cells and the transcript termination/polyadenylation (poly-A) sequence for mRNA transcript stabilization-with a prokaryotic vicinity that gives resolution and propagation in host bacteria. Once the DNA plasmid is administered by the route intradermal, intravenous, intraperitoneal, subcutaneous, nasal or intramuscular route, the plasmid is internalized into the host cells (myocytes and antigen-presenting cells), it translocates to the cellular nucleus where the host cell equipment initiates the transgenic transcription followed with the aid of the cytoplasmic translation of the transgenic into protein. For the use of DNA vaccine have no chances of risk of mutation with a viral vector. Therefore, it is more safety and efficacy related to others. DNA Vaccine does not require refrigeration for storage and the manufacturing process is easy.

\section{Micellar delivery}

Micelles are well identified and potential carriers for antigen delivery. Amphiphilic surfactant molecules are used to make the cluster of micelles. At the above CMC of surfactant, the structure of the micelles is keep away from the incompatible external phase. Micelles are surrounded by lipophilic or hydrophilic cavity; thus the transport of antigen in the body promoted by the antigen entrapment. It is the system in which one or more transport of vaccines via mucous improving agents like mixed micelles used as a carrier to expand manufacturing and bioavailability of the oral administration of antigen [45]. The various drug release mechanism involved through micellar delivery is presented in fig. 7.

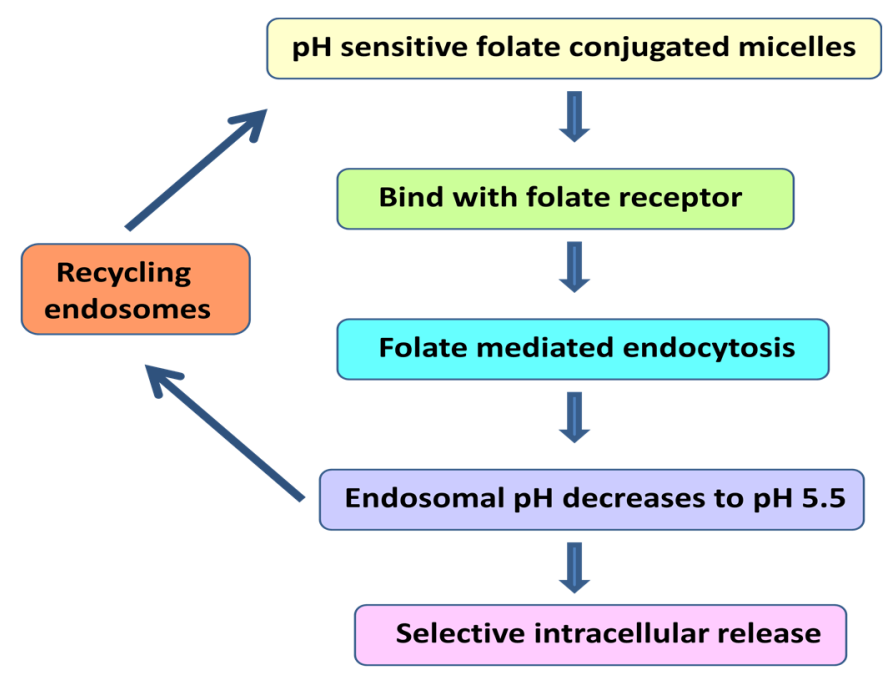

Fig. 7: Showing the mechanism of drug release from micelles

Some absorption enhancing agent is chosen from beta-cyclodextrin derivative or cyclodextrin mixed micelles surfactants. For the transdermal transport in development technique, a very useful agent is used, which are sensitive substances towards the temperature and $\mathrm{pH}$. In the aqueous solution, the temperaturesensitive substances are capable of producing salt to gel transition. In the temperature, a sensitive substance is a co-polymer (alkylene oxide), which is capable of producing micelles in aqueous solution. This type of formulation found to be properties of bio-adhesive and transport of many varieties of beneficial agents.

\section{Polymeric nanoparticles}

In recent years, a variety of antigens, proteins as well as vaccines for humans and animals use, have been encapsulated in 
microspheres/nanospheres. These are mainly composed of biodegradable polymer or PLGA. The encapsulated antigens were delivered to animals for mucosal immunization by oral or intranasal inoculations or injected by parenteral routes [6].

These are taken up by using the mucosal related lymphoid tissue because of the structure of polymeric nanoparticles. These are used for oral as well as for the nasal vaccine transport system. The highquality immunization can be achieved by the administration of these nano-adjuvants in a low dose of antigen antigens. These nanoadjuvant based nanoparticles suitable for oral transport of antigen due to their capability to release antigen. and protect the antigen from enzymatic degradation $[4,71]$. In the case of oral immunization of PLGA based nano-adjuvant nanoparticles improve the immunogenicity in rats. PLGA nanoparticles are suitable for vaccine transport because these are very slowly degradable. Monoclonal antibody (mAb) nanoparticles specific to M-cells and enhance the absorption of the immune response. EDTA like metal-chelating polymers based nano-adjuvant is used in controlled transport in vivo, due to the formation of non-covalent bonds with the epitopes. When nanospheres are administered by nasal route for prevention of RSV infection. Nanosphere increases the number of RSV specific antibodies such as IgA antibodies and cytotoxic T-cells (CT-cells) $[19,72]$.

\section{Edible vaccine}

This type of vaccines contains the macromolecules that will one epitope from other epitopes present on an antigen. Therefore such vaccines are safer in comparison to conventional vaccines. It provides useful effects to stimulate immunity by the use of live viruses or micro-organisms. The manufacturing of such vaccines is very magnificent. Manufacturing of vaccines from the plants or obtained naturally have more advantage than others. A natural vaccine from the plant origin is less costly and providing proteins. The storage of vaccines from the manufacturing to till use or administration in the host is required at cold temperature using refrigeration to maintain their shelf life. Mucosal and systemic immune response can be achieved by the administration of plant earned vaccines through the mucosal route $[23,45,46]$.

\section{CONCLUSION}

The enormous versatility of liposomes and the related peptides and virosomes endows them as highly valuable carrier systems for nanoadjuvants vaccine delivery. Besides improving antigen stability and presentation to immune-competent of cells, having specific properties including composition, size and surface properties, these nanocarriers also possess the ability to overcome biological barriers, such as skin and mucosa, and provide controlled and slow release of antigens.

\begin{tabular}{llll}
\hline ABBREVIATION & & \\
\hline APCs & Antigen-presenting cells & mAb & Monoclonal antibody \\
CMC & Critical micelle concentration & MAP & Multiple antigen peptide system \\
CMI & Cell-mediated immunity & MHC & Major histocompatibility complex \\
CMV & Cytomegalovirus & PLGA & Poly(lactic-co-glycolic acid) \\
DCs & dendritic cells & O/W & Oil in water \\
DNA & Deoxyribonucleic acid & O/W/O & Oil in water in oil \\
HAV & Hepatitis A virus & PNA & Peptide nucleic acid \\
HBV & hepatitis B virus & PPR & pattern recognition receptor \\
HIV & human immunodeficiency virus & TCR & Toll-like receptor \\
HPV & Human papilloma virus & TNF- $\alpha$ & Tumor necrosis factor-alpha \\
IFN & Interferon & SC & Subcutaneous \\
IL-2 & Interleukin & W/O & Water in oil \\
IM & Intramuscular & W/O/W & Water in oil in water \\
LNs & Lymph nodes & & \\
\hline
\end{tabular}

\section{FUNDING}

Nil

\section{AUTHORS CONTRIBUTIONS}

All authors have equally contributed

\section{CONFLICT OF INTERESTS}

No conflict of interest

\section{REFERENCES}

1. Ada G, Assaad FA, Blanden RV, Bloom BR, Brown F, Schild $\mathrm{GC}$, et al. New approaches to vaccine development: memorandum from a WHO meeting. Bull WHO 1985;63:479-84.

2. Tlaskalova Hogenova H, Tuckova L, Lodinova Zadnikova R, Stepankova R. Mucosal immunity: its role in defense and allergy. Int Arc Allergy Immunol 2002;128:77-9.

3. Reddy ST, Rehor A, Schmoekel HG, Hubbell JA, Swartz MA. In vivo targeting of dendritic cells in lymph nodes with poly(propylene sulfide) nanoparticles. J Controlled Release 2006;112:26-34.

4. Dineshkumar B, Dhanaraj SA, Santhi K, Vijayan P, Chandrasekhar R. Single-dose vaccine delivery system of tetanus toxoid formulation based on chitosan microspheres. Int J Adv Pharm Sci 2010;1:42-9.

5. Koutsonanos DG, del Pilar Martin M, Zarnitsyn VG, Sullivan SP, Compans RW, Prausnitz MR, et al. Transdermal influenza immunization with vaccine-coated microneedle arrays. Plos One 2009;4:4773-81.

6. Shankar SJ, Jasvanth Gowda BH, Akshatha RS, Basavaraj M, Mohamed R. A review on the role of nanocrystal and nanosuspension in drug delivery system. Int J Appl Pharm 2020;12:11-6.

7. Beck E, Strohmaier K. Subtyping of European foot-and-mouth disease virus strains by nucleotide sequence determination. J Virol 1987;61:1621-9.

8. Liau J, Prestidge CA, Hook S, Barnes TJ. Development of a multicompartmental oral vaccine delivery system. Eur J Pceutics Bio-Pceutics 2015;97:15-21.

9. Allen PM, Matsueda GR, Evans RJ, Dunbar Jr JB, Marshall GR, Unanue ER. Identification of the T-cell and Ia contact residues of a T-cell antigenic epitope. Nature 1987;327:713-22.

10. Hidaka A, Kiyono H, Kunisawa J, Aramaki Y. Intranasal administration of cationic liposomes enhanced granulocytemacrophage colony-stimulating factor expression and this expression is dispensable for mucosal adjuvant activity. BMC Res Notes 2018;11:472-83.

11. Dupuis M, McDonald DM, Ott G. Distribution of adjuvant MF59 and antigen gD2 after intramuscular injection in mice. Vaccine 1999;18:434-9.

12. Nicolas JF, Guy B. Intradermal, epidermal and transcutaneous vaccination: from immunology to clinical practice. Expert Rev Vaccines 2008;7:1201-4.

13. Schuch RA, Oliveira TL, Collares TF, Monte LG, RoigInda G, Dellagostin OA, et al. The use of xanthan gum as vaccine adjuvant: an evaluation of immunostimulatory potential in BALB/c mice and cytotoxicity in vitro. BioMed Res Int 2017;24:1-9.

14. Pasquale AD, Preiss S, Da Silva FT, Garçon N. Vaccine adjuvants: from 1920 to 2015 and beyond. Vaccines 2015;3:320-3.

15. Schwendener RA. Liposomes as vaccine delivery systems: a review of the recent advances. Expert Rev Vaccines 2014; 2:159-82. 
16. Borges O, Borchard G, Verhoef JC, de Sousa A, Junginger HE. Preparation of coated nanoparticles for a new mucosal vaccine delivery system. Int J Pharm 2005;299:155-6.

17. Arnon R. Synthetic peptides as the basis for future vaccines. Trends Biochem Sci 1986;11:521-4.

18. Arnon R, Shapira M, Jacob CO. Synthetic vaccines. J Immunol Methods 1983;61:261-73.

19. Atassi MZ, Casali P, Atassi MZ, Casali P. Molecular mechanisms of autoimmunity. Autoimmunity 1988;41:123-32.

20. Bachmann MF, Jennings GT. Vaccine delivery: a matter of size, geometry, kinetics and molecular patterns. Nat Rev Immunol 2010;10:787-96.

21. Ada GL. What to expect of a good vaccine and how to achieve it. Vaccine 1988;6:77-9.

22. Broecker F, Gotze S, Hudon J, Dominea CK, Pereira CL, Stallforth $\mathrm{P}$, et al. Synthesis, liposomal formulation, and immunological evaluation of a minimalistic carbohydrate- $\alpha$-galcer vaccine candidate. J Med Chem 2018;61:4918-27.

23. Smirnov D, Schmidt JJ, Capecchi JT, Wightman PD. Vaccine adjuvant activity of 3M-052: an imidazoquinoline designed for local activity without systemic cytokine induction. Vaccine 2011;29:5434-42.

24. Shi S, Hickey AJ. PLGA microparticles in respirable sizes enhance an in vitro $\mathrm{T}$ cell response to recombinant mycobacterium tuberculosis antigen TB10. 4-Ag 85B. Pharm Res 2010;27:350-60.

25. Pulendran B, Ahmed R. Immunological mechanisms of vaccination. Nat Immunol 2011;12:509-17.

26. Moon JJ, Suh H, Li AV, Ockenhouse CF, Yadava A. Enhancing humoral responses to a malaria antigen with nanoparticle vaccines that expand Tfh cells and promote germinal center induction. Proc Natl Acad Sci USA 2012;109:1080-5.

27. Kumar P, Nene AG, Punia S, Kumar M, Abbas Z. Synthesis, characterization and antibacterial activity of cuo nanoparticles. Int J Appl Pharm 2020;12:17-20.

28. Mohapatra SS. Mucosal gene expression vaccine: a novel strategy for respiratory syncytial virus. Pediatr Infect Dis 2003;21:S100-3.

29. Hubbell JA, Thomas SN, Swartz MA. Materials engineering for immunomodulation. Nature 2009;462:449-60.

30. Pardakhty A, Moazeni E. Nano-niosomes in drug, vaccine and gene delivery: a rapid overview. Nanomedicine 2013;1:1-12.

31. Rathore P, Swami G. Virosomes: a novel vaccination technology. Int J Pharm Sci Res 2012;3:3591-7.

32. Amselem S, Alving CR, Domb AJ. Polymeric biodegradable lipospheres ${ }^{\mathrm{TM}}$ as vaccine delivery systems. Polym Adv Technol 1993;3:351-7.

33. Mohapatra SS. Mucosal gene expression vaccine: a novel vaccine strategy for the respiratory syncytial virus. Pediatric Infectious Disease J 2003;22:S100-S104.

34. Zaks K, Jordan M, Guth A, Sellins K, Kedl R, Izzo A, Dow S. Efficient immunization and cross-priming by vaccine adjuvants containing TLR3 or TLR9 agonists complexed to cationic liposomes. J Immunol 2006;176:7335-45.

35. Aiswarya MU, Keerthana R, Revathy BM, Lakshmi VS, Sreeja CN Cryptosomes: a revolutionary breakthrough in novel drug delivery. Int J Appl Pharm 2019;11:7-13.

36. Sporri R, Reis-e-Sousa C. Inflammatory mediators are insufficient for full dendritic cell activation and promote the expansion of CD4+T cell populations lacking helper function. Nat Immunol 2005;6:163-70.

37. Supersaxo A, Hein WR, Steffen H. Effect of molecular weight on the lymphatic absorption of water-soluble compounds following subcutaneous administration. Pharm Res 1990; 7:167-9.

38. Ali AA, McCrudden CM, McCaffrey J, McBride JW, Cole G, Dunne $\mathrm{NJ}$, et al. DNA vaccination for cervical cancer; a novel technology platform of RALA mediated gene delivery via polymeric microneedles. Nanomed: Nanotechnol Biol Med 2017;13:921-32.

39. Mildner A, Jung S. Development and function of dendritic cell subsets. Immunity 2014;40:642-6.
40. O'Hagan DT, Ott GS, De Gregorio E, Seubert A. The mechanism of action of MF59-an innately attractive adjuvant formulation. Vaccine 2012;30:4341-8.

41. Cabral H, Kotaoka K. Multifunctional nano assembles of block copolymers for future cancer therapy. Sci Technol Adv Mater 2010;10:1-9.

42. Garçon N, Chomez P, Van Mechelen M. Glaxo-smithkline adjuvant systems in vaccines: concepts, achievements and perspectives. Expert Rev Vaccines 2007;6:723-9.

43. Cabral H, Kataoka K. Multifunctional nanoassemblies of block copolymers for future cancer therapy. Sci Technol Adv Mater 2010;11:104-13.

44. Tacken PJ, de Vries IJM, Torensma R, Figdor CG. Dendritic-cell immunotherapy: from ex vivo loading to in vivo targeting. Nat Rev Immunol 2007;7:790-802.

45. Luo M, Samandi LZ, Wang Z, Chen ZJ, Gao J. Synthetic nanovaccines for immunotherapy.J Controlled Release 2017;263:200-10.

46. Qu W, Li N, Yu R, Zuo W, Fu W. Cationic DDA/TDB liposome as a mucosal vaccine adjuvant for uptake by dendritic cells in vitro induces potent humoural immunity. Artif Cells Nanomed Biotechnol 2018;46:S852-S960.

47. Ssemaganda A, Giddam AK, Zaman M, Skwarczynski M, Toth I, Stanisic DI, et al. Induction of plasmodium-specific immune responses using liposome-based vaccines. Front Immunol 2019;10:231-42.

48. Manolova V, Flace A, Bauer M, Schwarz K, Saudan P, et al. Nanoparticles target distinct dendritic cell populations according to their size. Eur J Immunol 2008;38:1404-13.

49. Joshi VB, Geary SM, Salem AK. Biodegradable particles as vaccine antigen delivery systems for stimulating cellular immune responses. Hum Vaccines Immunother 2013;9:258490.

50. Da Costa Martins R, Gamazo C, Sanchez Martinez M, Barberan $\mathrm{M}$, Penuelas I. Conjunctival vaccination against Brucella ovis in mice with mannosylated nanoparticles. J Controlled Release 2012;162:553-60.

51. Chackerian B, Lowy DR, Schiller JT. Conjugation of a selfantigen to papillomavirus-like particles allows for efficient induction of protective. J Clin Invest 2001;108:415-23.

52. Pasare C, Medzhitov R. Control of B-cell responses by Toll-like receptors. Nature 2005;438:364-8.

53. Karshalm KS, Anderson P, Peril Y, Christenseen D. Liposomal vaccine delivery. Drug Delivery 2011;8:505-19.

54. Joshi M, Lata S, Kanwar P, Mishra T. Application of nanostructures in antimicrobial therapy. Int J Appl Pharm 2018;18:11-25.

55. Pardakhty A, Moazenil E. Nano-niosomes in drug vaccine and gene delivery: a rapid overview. Nanomedicine 2012;1:1-6.

56. Link A, Zabel F, Schnetzler Y, Titz A, Brombacher F, et al. Innate immunity mediates follicular transport of particulate but not soluble protein antigen. J Immunol 2012;188:3724-33.

57. Pintar A, Carugo O, Pongor S. CX, an algorithm that identifies protruding atoms in proteins. Bioinformatics 2005;18:980-4.

58. Donnelly RF. Vaccine delivery systems. Hum Vaccines Immunother 2017;13:17-26.

59. Carino GP. Vaccine delivery. Mathiowitz E. editor. Encyclopedia of Controlled. Drug Delivery 1999;4:996-7.

60. Dhakal S, Cheng X, Salcido J, Renu S, Bondra K, Lakshmanappa YS, et al. Liposomal nanoparticle-based conserved peptide influenza vaccine and monosodium urate crystal adjuvant elicit a protective immune response in pigs. Int J Nanomed 2018;13:6699-15.

61. Oyewumi MO, Kumar A, Cui Z. Nano-microparticles as immune adjuvants: correlating particle sizes and the resultant immune responses. Expert Rev Vaccines 2010;9:1095-107.

62. Lee S, Nguyen MT. Recent advances of vaccine adjuvants for infectious diseases. Immune Network 2015;15:51-7.

63. Sexton A, Whitney PG, Chong SF, Zelikin AN, Johnston AP. A protective vaccine delivery system for in vivo $\mathrm{T}$ cell stimulation using nanoengineered polymer hydrogel capsules. Acs Nano 2009;3:3391-400.

64. Bekiaris V, Persson EK, Agace WW. Intestinal dendritic cells in the regulation of mucosal immunity. Immunol Rev 2014;12:86-101. 
65. Kato LM, Kawamoto S, Maruya M, Fagarasan S. The role of the adaptive immune system in regulation of gut microbiota. Immunol Rev 2014;21:67-75.

66. Kalra N, Dhanya V, Saini V, Jeyabalan G. Virosomes: as a drug delivery carrier. Am J Adv Drug Delivery 2013;1:29-35.

67. Napolitani G, Rinaldi A, Bertoni F, Sallusto F, Lanzavecchia A. Selected toll-like receptor agonist combinations synergistically trigger a T helper type 1-polarizing program in dendritic cells. Nat Immunol 2005;6:769-76.

68. Caminschi I, Shortman K. Boosting antibody responses by targeting antigens to dendritic cells. Trends Immunol 2012;33:71-7.
69. Moyer TJ, Zmolek AC, Irvine DJ. Beyond antigens and adjuvants: formulating future vaccines. J Clin Invest 2016;126:7999-808.

70. Gupta RK, Chang AC, Siber GR. Biodegradable polymer microspheres as vaccine adjuvants and delivery systems. Dev Biol Stand 1998;92:63-8.

71. Ramamurthy SK, Shidhar C. Parthenium mediated synthesis of zinc oxide nanoparticles and its characterization. Int J Appl Pharm 2019;11:113-6.

72. Arnon R. Peptides as immunogens: prospects for synthetic vaccines. In Peptides as Immunogens. Springer Berlin Heidelberg 1986;5:1-2. 